\title{
Nonclassicality Criteria in Multiport Interferometry
}

\author{
L. Rigovacca, ${ }^{1, *}$ C. Di Franco, ${ }^{1,2,3}$ B. J. Metcalf, ${ }^{4}$ I. A. Walmsley, ${ }^{4}$ and M. S. Kim ${ }^{1}$ \\ ${ }^{1}$ Blackett Laboratory, Imperial College London, London SW7 2AZ, United Kingdom \\ ${ }^{2}$ School of Physical and Mathematical Sciences, Nanyang Technological University, 637371, Singapore \\ ${ }^{3}$ Complexity Institute, Nanyang Technological University, 637723, Singapore \\ ${ }^{4}$ Clarendon Laboratory, University of Oxford, Parks Road, Oxford OX1 3PU, United Kingdom
}

(Received 29 April 2016; published 16 November 2016)

\begin{abstract}
Interference lies at the heart of the behavior of classical and quantum light. It is thus crucial to understand the boundaries between which interference patterns can be explained by a classical electromagnetic description of light and which, on the other hand, can only be understood with a proper quantum mechanical approach. While the case of two-mode interference has received a lot of attention, the multimode case has not yet been fully explored. Here we study a general scenario of intensity interferometry: we derive a bound on the average correlations between pairs of output intensities for the classical wavelike model of light, and we show how it can be violated in a quantum framework. As a consequence, this violation acts as a nonclassicality witness, able to detect the presence of sources with sub-Poissonian photon-number statistics. We also develop a criterion that can certify the impossibility of dividing a given interferometer into two independent subblocks.
\end{abstract}

DOI: 10.1103/PhysRevLett.117.213602

Hong, $\mathrm{Ou}$, and Mandel (HOM) discovered that if two independent and indistinguishable photons, in pure quantum states, impinge on the two input ports of a balanced beam splitter, they always bunch together and exit the apparatus from the same output port [1]. This simple effect has many consequences, e.g., in distinguishability testing [2], linear-optical quantum computing [3], entanglement detection [4] or swapping [5], and metrology [6-9]. The nonclassicality of this phenomenon can be well understood by repeating the experiment many times, and by recording the intensities $I_{1}, I_{2}$ at the two output ports: labeling the average over many runs by $\langle\cdot\rangle$, the correlation function,

$$
G_{12}=\frac{\left\langle I_{1} I_{2}\right\rangle}{\left\langle I_{1}\right\rangle\left\langle I_{2}\right\rangle},
$$

will be zero in the ideal case, because on each run the intensity at one of the two ports will vanish. $G_{12}$ has a well-defined classical limit, which makes it a suitable candidate to use in distinguishing quantum light beams from classical ones. We can either consider completely distinguishable photons, i.e., single excitations occupying orthogonal space-time modes, or pulses of classical light, described by electromagnetic fields. In both cases, if they are emitted by statistically independent sources and injected into the beam splitter, $G_{12}$ is constrained to be greater than or equal to $1 / 2[10,11]$. Therefore, the value $G_{12}=0$ obtained in the ideal HOM effect represents a strong signature of nonclassicality.

Published by the American Physical Society under the terms of the Creative Commons Attribution 3.0 License. Further distribution of this work must maintain attribution to the author(s) and the published article's title, journal citation, and DOI.
Several authors have investigated interference effects of noninteracting particles with the aim of reproducing or generalizing HOM's result to different situations (see Refs. [12,13]) not necessarily constrained to linear optics [14-18]. However, photonics remains the physical platform of choice for these studies, since it is now possible to prepare and manipulate several photons in ambient laboratory conditions, which can then be injected into multimode interferometers [19-26]. The recent investigations of many-particle interference effects have revealed a need for a deeper understanding of the phenomenon. On the computational side, boson sampling is a feasible candidate to show the possibility of outperforming classical computers exploiting the laws of quantum mechanics [23,24,27-31]. From a foundational perspective, on the other hand, the interplay between the wavelike behavior of photons and the manyparticle interference effects arising due to their bosonic nature is not well understood [13,32-37]. This is an important issue because these two features heavily influence the probabilities of detection events, often leading to counterintuitive results [32,36,37]. Typically, these studies compare their findings with the evolution of completely distinguishable photons; this retains the quantization of the number of particles, but removes interparticle interference.

In this Letter we make the complementary choice, by studying the alternative classical regime where independent sources emit light pulses fully described in terms of their electric field. As already mentioned, in a situation with two sources and two detectors, the classical bound $G_{12} \geq 1 / 2$ holds, which can be maximally violated by the HOM setup, i.e., $G_{12}=0$. It is, therefore, natural to ask how this result can be extended to a more general framework, with an arbitrary number of sources and detectors. Here we provide 
an answer to this question, by finding a tight lower bound for the correlations that can arise among the output intensities of a generic multiport interferometer, when the aforementioned classical sources are used. By using a quantum mechanical approach, we then study "if" and "by how much" quantum input states of light can violate this threshold. Finally, we show how our findings allow us to develop a sufficient criterion that can certify the impossibility of dividing an interferometer into independent subblocks.

Correlation function.--In characterizing the correlations among several detected intensities, say, $M \geq 2$, we have to choose a generalization of $G_{12}$, defined in Eq. (1). Recently, several authors have considered higher-order correlation functions (i.e., in which the average involves products of more than two intensities) to study multiparticle interference $[34,38]$ or to obtain advantages in imaging resolution [39-42]. On the other hand, Walschaers et al. showed that the simpler quantity $\left\langle I_{i} I_{j}\right\rangle-\left\langle I_{i}\right\rangle\left\langle I_{j}\right\rangle$ can yield information on the statistics of the interfering particles [43] and on their distinguishability [44] if it is averaged over many output modes of a generic interferometer. We introduce a similar but slightly different quantifier, obtained as the normalized average,

$$
\bar{G}=\frac{1}{\left(\begin{array}{c}
M \\
2
\end{array}\right)} \sum_{i<j} \frac{\left\langle I_{i} I_{j}\right\rangle}{\left\langle I_{i}\right\rangle\left\langle I_{j}\right\rangle},
$$

where $i<j$ enforces the sum to be over all pairs of detectors. The normalization chosen in Eq. (2) assures that $\bar{G}$ does not depend on the average intensities, but only on their correlations: this is a necessary condition to obtain a classical bound independent of the total intensities of the sources. With respect to considering higher-order correlators, $\bar{G}$ has the advantage of being composed of many simpler contributions, while still taking into account all available data. Moreover, the measure of $\bar{G}$ only requires the simultaneous intensity readouts in two detectors, thus allowing a quicker experimental estimation in the presence of detector inefficiencies. We also point out that the experimental effort required to estimate $\left\langle I_{i} I_{j}\right\rangle$, and therefore $\bar{G}$, has a polynomial scaling with the number of sources and photon-number resolving detectors [43]. Finally, note that only detectors with $\left\langle I_{i}\right\rangle \neq 0$ (i.e., receiving a nonzero amount of light) should be considered in the average of Eq. (2), in order to keep $\bar{G}$ well defined.

Description of the setup.-We now describe the interferometric setup, sketched in Fig. 1, in the classical and quantum scenario. In both cases pulses of light are emitted by $N$ sources and are detected by $M$ detectors after a linear evolution. As in the HOM setup, in each realization the phases of the pulses are chosen randomly in $[0,2 \pi]$, the sources are independent, and are allowed to be stochastic. More precisely, at every run of the experiment in the classical case the $\alpha$ th source emits the electric field $\vec{E}_{\alpha, \xi_{\alpha}}(t)=A_{\alpha}\left(\xi_{\alpha}\right) \vec{\zeta}^{\left(\xi_{\alpha}\right)}(t)$ with probability $p_{\alpha}\left(\xi_{\alpha}\right)$. Here

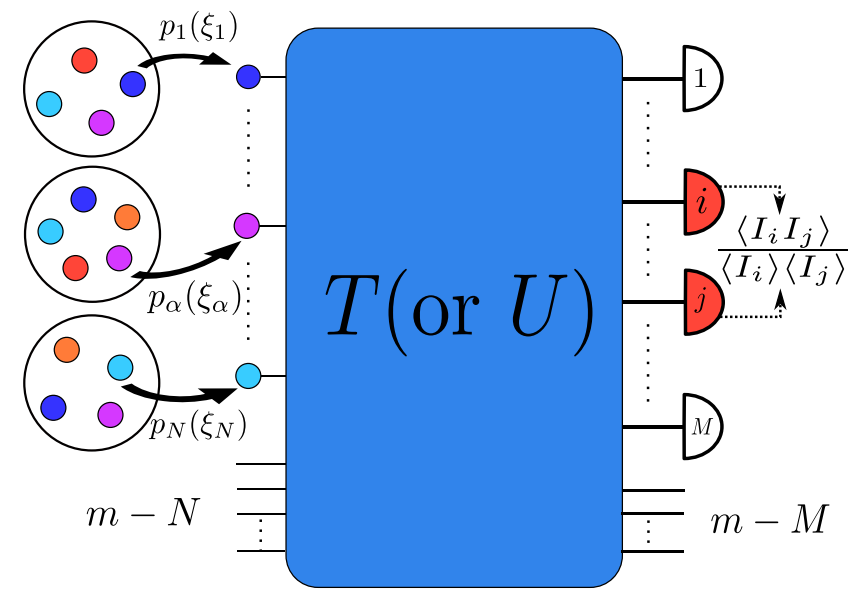

FIG. 1. Sketch of the setup described in the main text, with $N$ sources and $M$ detectors. In the classical (quantum) picture the interferometer is characterized by the transfer matrix $T(U)$, while the colored circles represent the light pulses $\vec{E}_{\alpha, \xi_{\alpha}}(t)$ (the states $\left.\left|\varphi_{\alpha}^{\left(\xi_{\alpha}\right)}\right\rangle\right)$. In the quantum framework the number $m$ of interferometric modes has to be greater than $N$ and $M$ : if the inequalities are strict there will be vacuum inputs and nonmonitored outputs. If at least two detections are successful, the event can be used for the evaluation of $\bar{G}$.

$A_{\alpha}\left(\xi_{\alpha}\right)$ is a complex number whose phase changes randomly from one pulse to the other, while $\vec{\zeta}_{\alpha}^{\left(\xi_{\alpha}\right)}(t)$ defines the mode of the field. In particular, if $\left\{\vec{\epsilon}_{\omega, \lambda}\right\}_{\lambda}$ are orthonormal polarization vectors, one has

$$
\vec{\zeta}_{\alpha}^{\left(\xi_{\alpha}\right)}(t)=\sum_{\lambda} \int d \omega \sqrt{\frac{\hbar \omega}{2 \pi}} g_{\omega, \lambda}\left(\xi_{\alpha}\right) e^{-i \omega t} \vec{\epsilon}_{\omega, \lambda}
$$

where the coefficients $\left\{g_{\omega, \lambda}\left(\xi_{\alpha}\right)\right\}$ depend on the value of the random variable $\xi_{\alpha}$ and satisfy the relation $\sum_{\lambda} \int d \omega\left|g_{\omega, \lambda}\left(\xi_{\alpha}\right)\right|^{2}=1$. We added the factor $\hbar \omega$ only to ease the comparison with the quantum case later on, but classically it could be included in $g_{\omega, \lambda}$ and $A_{\alpha}$. The linear evolution can then be represented via a complex transfer matrix $T$, which maps the input fields $\left\{\vec{E}_{\alpha, \xi_{\alpha}}\right\}$ to those at the detectors' positions, labeled by $i=1, \ldots, M$ :

$$
\vec{O}_{\overrightarrow{i, \vec{\xi}}}(t)=\sum_{\alpha=1}^{N} T_{i \alpha} \vec{E}_{\alpha, \xi_{\alpha}}\left(t-\tau_{i \alpha}\right),
$$

where $\tau_{i \alpha}$ are the evolution times and $\vec{\xi}=\left(\xi_{1}, \ldots, \xi_{N}\right)$. The intensity measured by the $i$ th detector will therefore be, up to a dimensional proportionality factor,

$$
I_{i, \vec{\xi}}=\int_{-\tau_{M} / 2}^{+\tau_{M} / 2} d t \vec{O}_{i, \vec{\xi}}^{*}(t) \cdot \vec{O}_{i, \vec{\xi}}(t),
$$

where we consider the measurement time $\tau_{M}$ to be much longer than the other time scales, so that the integration can 
be equivalently performed over the whole real axis. This means that the detectors measure a quantity proportional to the energy, or integrated flux, of the light pulses. The quantities $\left\langle I_{i}\right\rangle$ and $\left\langle I_{i} I_{j}\right\rangle$ are then obtained by averaging over the configurations $\{\vec{\xi}\}$. Note that their evaluation is simplified by the following relations imposed by the randomness of the phase characterizing each pulse of light: $\left\langle A_{\alpha}\right\rangle=\left\langle A_{\alpha} A_{\beta}\right\rangle=0$ and $\left\langle A_{\alpha}^{*} A_{\beta}\right\rangle=\delta_{\alpha, \beta}\left\langle\left|A_{\alpha}\right|^{2}\right\rangle$.

In order to move to a quantum picture, an operator for the electric field emitted by each source is defined as

$$
\hat{E}_{\alpha}(t)=\sum_{\lambda} \int d \omega \sqrt{\frac{\hbar \omega}{2 \pi}} \hat{a}_{\alpha ; \omega, \lambda} e^{-i \omega t} \vec{\epsilon}_{\omega, \lambda},
$$

up to an irrelevant factor, where the bosonic annihilation operators $\left\{\hat{a}_{\alpha ; \omega, \lambda}\right\}$ satisfy the canonical commutation relations $\left[\hat{a}_{\alpha ; \omega, \lambda}, \hat{a}_{\beta ; \omega^{\prime}, \lambda^{\prime}}^{\dagger}\right]=\delta_{\alpha, \beta} \delta_{\lambda, \lambda^{\prime}} \delta\left(\omega-\omega^{\prime}\right)$ (see Ref. [45]). With probability $p_{\alpha}\left(\xi_{\alpha}\right)$, the $\alpha$ th source then emits the quantum state

$$
\left|\varphi_{\alpha}^{\left(\xi_{\alpha}\right)}\right\rangle=\sum_{n=0}^{\infty} \varphi_{\alpha}^{\left(\xi_{\alpha}\right)}(n)\left(\hat{a}_{\alpha ; \xi_{\alpha}}^{\dagger}\right)^{n}|0\rangle,
$$

where the amplitudes $\left\{\varphi_{\alpha}^{\left(\xi_{\alpha}\right)}(n)\right\}_{n}$ depend on the random variable $\xi_{\alpha},|0\rangle$ represents the vacuum, and $\hat{a}_{\alpha ; \xi_{\alpha}}$ can be decomposed as

$$
\hat{a}_{\alpha ; \xi_{\alpha}}=\sum_{\lambda} \int d \omega g_{\omega, \lambda}^{*}\left(\xi_{\alpha}\right) \hat{a}_{\alpha ; \omega, \lambda}
$$

However, note that the coherence between different photon numbers in Eq. (7) is lost once we average over many realizations of the same pulse, because each of them is emitted with a random phase multiplying $g_{\omega, \lambda}^{*}$ in Eq. (8). In this scenario, it is natural to inject the emitted states in an $m \times m$ linear optical interferometer, with $m \geq M, N$. For any given realization $\vec{\xi}$ of the sources, the output modes will be characterized by the set of operators $\left\{\hat{b}_{i ; \vec{\xi}}\right\}_{i=1}^{m}$, obtained as $\hat{b}_{i, \vec{\xi}}=\sum_{\alpha=1}^{m} U_{i \alpha} \hat{a}_{\alpha ; \xi_{\alpha}}$, where $U$ is a unitary matrix that plays a role analogous to the classical $T$. The intensities appearing in Eq. (2) are obtained, up to a dimensional proportionality factor, by taking the expectation value of the operators $\hat{I}_{i}$ and $\hat{I}_{i} \hat{I}_{j}$ on the input state, where

$\hat{I}_{i}=\int_{-\tau_{M} / 2}^{+\tau_{M} / 2} d t \hat{E}_{i}^{\dagger}(t) \hat{E}_{i}(t)=\sum_{\lambda} \int d \omega \hat{b}_{i ; \omega, \lambda}^{\dagger} \hat{b}_{i ; \omega, \lambda} \hbar \omega$.

For the sake of simplicity, in the following we will assume that the mode of the emitted fields is characterized by the same weights $g_{\omega, \lambda}\left(\xi_{0}\right)$ for all sources and all realizations, and that the evolution times $\tau_{i \alpha}$ are all the same. Intuitively, these conditions maximize the interference and in the classical case lead to the minimum value of Eq. (2) (see Ref. [46] for the proof). With these hypotheses, it turns out that the averaged intensities that appear in the classical or quantum expression of $\bar{G}$ are proportional to $\mathcal{E}=\sum_{\lambda} \int d \omega \hbar \omega\left|g_{\omega, \lambda}\left(\xi_{0}\right)\right|^{2}$ [46], which represents the energy associated with the chosen mode. In particular, classically one has $I_{i, \vec{\xi}}=\mathcal{E} A_{i}(\vec{\xi})^{*} A_{i}(\vec{\xi})$, where $A_{i}(\vec{\xi})=$ $\sum_{\alpha=1}^{N} T_{i_{\alpha}} A_{\alpha}\left(\xi_{\alpha}\right)$, while in the quantum case, one finds

$$
\begin{gathered}
\left\langle\hat{I}_{i}\right\rangle=\mathcal{E} \operatorname{Tr}\left[\hat{\rho} \hat{b}_{i ; \xi_{0}}^{\dagger} \hat{b}_{i ; \xi_{0}}\right], \\
\left\langle\hat{I}_{i} \hat{I}_{j}\right\rangle=\mathcal{E}^{2} \operatorname{Tr}\left[\hat{\rho} \hat{b}_{i ; \xi_{0}}^{\dagger} \hat{b}_{j ; \xi_{0}}^{\dagger} \hat{b}_{i ; \xi_{0}} \hat{b}_{j ; \xi_{0}}\right] .
\end{gathered}
$$

This is intuitive because the intensity of the quantum field is directly connected with the photon number, when each photon carries the same amount of energy. In Eqs. (10) and (11), $\hat{\rho}$ is the average emitted state,

$$
\hat{\rho}=\bigotimes_{\alpha=1}^{N} \sum_{n_{\alpha}} \frac{q_{\alpha}\left(n_{\alpha}\right)}{n_{\alpha} !}\left(\hat{a}_{\alpha, \xi_{0}}^{\dagger}\right)^{n_{\alpha}}|0\rangle\langle 0|\left(\hat{a}_{\alpha, \xi_{0}}\right)^{n_{\alpha}},
$$

where $q(\vec{n})=q_{1}\left(n_{1}\right) \ldots q_{N}\left(n_{N}\right)$ is the effective probability distribution of the process [47]. In the following, when Eq. (2) is calculated in the classical or quantum regime, it will be written, respectively, as $\bar{G}^{(\mathrm{cl})}$ or $\bar{G}^{(Q)}$. We will also drop the label $\xi_{0}$ from the bosonic operators.

Classical bound.-We now look for the minimum value that $\bar{G}^{(\mathrm{cl})}$ can take. This will be the benchmark against which the results of an experiment must be compared in order to certify a nonclassical behavior, i.e., the impossibility of simulating the same result with only classical resources. Explicit calculations yield $\left\langle I_{i}\right\rangle=\mathcal{E} \sum_{\alpha=1}^{N}\left|T_{i \alpha}\right|^{2}\left\langle\left|A_{\alpha}\right|^{2}\right\rangle$ and

$$
\begin{aligned}
\left\langle I_{i} I_{j}\right\rangle= & \left\langle I_{i}\right\rangle\left\langle I_{j}\right\rangle+\mathcal{E}^{2} \sum_{\alpha \neq \beta}^{N} T_{i \alpha} T_{i \beta}^{*} T_{j \beta} T_{j \alpha}^{*}\left\langle\left|A_{\alpha}\right|^{2}\right\rangle\left\langle\left|A_{\beta}\right|^{2}\right\rangle \\
& +\mathcal{E}^{2} \sum_{\alpha=1}^{N}\left|T_{i \alpha}\right|^{2}\left|T_{j \alpha}\right|^{2}\left[\left\langle\left|A_{\alpha}\right|^{4}\right\rangle-\left\langle\left|A_{\alpha}\right|^{2}\right\rangle^{2}\right],
\end{aligned}
$$

where the last (positive) term vanishes for sources with fixed intensity, which are, therefore, optimal. The remaining minimization can be performed by defining a set of $M$ normalized vectors $\psi_{i} \in \mathbb{C}^{N}$ with components $\psi_{i}(\alpha)=$ $T_{i \alpha}^{*} \sqrt{\mathcal{E}\left\langle\left|A_{\alpha}\right|^{2}\right\rangle /\left\langle I_{i}\right\rangle}$, which allow us to rewrite $\bar{G}^{\text {(cl) }}$ as

$\bar{G}^{(\mathrm{cl})}=1+\frac{1}{\left(\begin{array}{c}M \\ 2\end{array}\right)} \sum_{i<j}^{M}\left(\left|\psi_{i}^{*} \cdot \psi_{j}\right|^{2}-\sum_{\alpha=1}^{N}\left|\psi_{i}(\alpha) \psi_{j}(\alpha)\right|^{2}\right)$,

where $\psi_{i}^{*} \cdot \psi_{j}=\sum_{\alpha} \psi_{i}^{*}(\alpha) \cdot \psi_{j}(\alpha)$. This expression can be minimized with respect to the vectors $\psi_{i}$ (see the Appendix), yielding

$$
\min \bar{G}_{N, M}^{(\mathrm{cl})}= \begin{cases}1-\frac{N-1}{N(M-1)} & \text { if } N \leq M \\ 1-\frac{1}{M} & \text { if } N \geq M,\end{cases}
$$


where the subscripts on the left-hand side emphasize the dependence on the number of sources and detectors (respectively, $N$ and $M$ ). We can verify that the minimum is reached by letting light fields with the same input intensity evolve with a highly symmetric $T$ : the $M \times M$ Fourier transform matrix (FTM), whose $(j, \alpha)$ element is given by $e^{2 \pi i(j-1)(\alpha-1) / M} / \sqrt{M}$. Intuitively, this interferometric apparatus yields the minimum output correlations because it leads to a high degree of interference: the input intensities are equally split among all outputs and the phases are symmetrically distributed over $2 \pi$. We point out that, when $N>M$, the configuration that achieves the $1-1 / M$ bound completely ignores $N-M$ sources (see the Appendix), whose light fields never reach the detectors. This setup is, therefore, effectively equivalent to a symmetric one smaller in size, with only $M$ sources and detectors.

Quantum description.-The explicit evaluation of Eqs. (10) and (11) follows the steps of other studies (see Refs. $[34,43,44])$, and for the input state in Eq. (12), one finds $\left\langle\hat{I}_{i}\right\rangle=\mathcal{E} \sum_{\alpha=1}^{m}\left|U_{i \alpha}\right|^{2}\left\langle\hat{n}_{\alpha}\right\rangle_{q}$ and

$$
\begin{aligned}
\left\langle\hat{I}_{i} \hat{I}_{j}\right\rangle= & \left\langle\hat{I}_{i}\right\rangle\left\langle\hat{I}_{j}\right\rangle+\mathcal{E}^{2} \sum_{\alpha \neq \beta}^{m} U_{i \alpha} U_{i \beta}^{*} U_{j \beta} U_{j \alpha}^{*}\left\langle\hat{n}_{\alpha}\right\rangle_{q}\left\langle\hat{n}_{\beta}\right\rangle_{q} \\
& +\mathcal{E}^{2} \sum_{\alpha=1}^{m}\left|U_{i \alpha}\right|^{2}\left|U_{j \alpha}\right|^{2}\left[\left(\left\langle\hat{n}_{\alpha}^{2}\right\rangle_{q}-\left\langle\hat{n}_{\alpha}\right\rangle_{q}^{2}\right)-\left\langle\hat{n}_{\alpha}\right\rangle_{q}\right],
\end{aligned}
$$

where $\hat{n}_{\alpha}=\hat{a}_{\alpha}^{\dagger} \hat{a}_{\alpha}$ and the subscript $q$ reminds us of the effective probability distribution appearing in Eq. (12). Note that if there are more interferometric modes than sources (i.e., $N<m$ ), we can trivially extend the definition of $\hat{\rho}$ in Eq. (12) by considering $q_{\alpha>N}\left(n_{\alpha}\right)=\delta_{n_{\alpha}, 0}$. Apart from the natural correspondences $U \leftrightarrow T$ and $\left\langle\hat{n}_{\alpha}\right\rangle_{q} \leftrightarrow\left\langle\left|A_{\alpha}\right|^{2}\right\rangle$, we can see how the main difference between the classical and the quantum quantities lies in the presence of a negative term linear in $\hat{n}_{\alpha}$ in Eq. (16). Its origin is a direct consequence of the photon-number quantization via the relation $\left\langle n\left|\hat{a}^{\dagger 2} \hat{a}^{2}\right| n\right\rangle=n(n-1)$. This immediately shows that a necessary condition to observe a violation of the bound in Eq. (15) is that the effective photon-number statistics has to be sub-Poissonian for some source; i.e.,

$$
\exists \alpha:\left\langle\hat{n}_{\alpha}^{2}\right\rangle_{q}-\left\langle\hat{n}_{\alpha}\right\rangle_{q}^{2} \leq\left\langle\hat{n}_{\alpha}\right\rangle_{q}
$$

For example, as the squeezed vacuum is super-Poissonian, this is immediately excluded from violating Eq. (15), despite being considered nonclassical in other situations. The condition in Eq. (17), however, is not sufficient to guarantee values of $\bar{G}^{(Q)}$ smaller than the classical threshold, because other inputs might have large intensity fluctuations which prevent the bound from being violated. On the other hand, similarly to the two-mode case [48], a single sub-Poissonian source could be sufficient to violate Eq. (15), for example, when coherent states with the same average intensity as the tested input are injected in all other ports. As could be expected, in the particular case in which all quantum sources emit coherent states (with phases randomly chosen) of amplitudes $\gamma_{\alpha}$, Eq. (16) reduces to Eq. (13), with $\gamma_{\alpha}$ playing the role of $A_{\alpha}$.

After having shown that the violation of the bound in Eq. (15) is possible for certain nonclassical states of light, we now study to what extent this threshold could be beaten. The presence in Eq. (16) of a term that is linear in the number of photons makes the minimization of $\bar{G}^{(Q)}$ considerably harder than its classical counterpart. However, an analytical minimum can be found at least in the symmetric case where states with the same subPoissonian photon-number statistics $q(n)$ are injected in every input port (situation labeled by "sym"). Although not general, this case is of interest in the study of many-particle interference effects, where the presence of vacuum inputs is not required as it would be in boson sampling. A symmetric setup allows a more intuitive and balanced picture and has been the study of several investigations (see Refs. $[12,13,21])$. In this case, all the information on the input statistics is given by

$$
0 \leq \eta(q)=-\frac{\left\langle\hat{n}^{2}\right\rangle_{q}-\langle\hat{n}\rangle_{q}^{2}-\langle\hat{n}\rangle_{q}}{\langle\hat{n}\rangle_{q}^{2}} \leq 1,
$$

whose positivity signals sub-Poissonian input states while its maximum value of 1 is reached for single-photon sources. With an approach analogous to the classical case, we can define the complex vectors $\tilde{\psi}_{i} \in \mathbb{C}^{N=m}$ with components $\tilde{\psi}_{i}(\alpha)=U_{i \alpha}^{*} \sqrt{\mathcal{E}\left\langle\hat{n}_{\alpha}\right\rangle_{q} /\left\langle\hat{I}_{i}\right\rangle}$. The output correlations measured by $\bar{G}$ become then

$\bar{G}_{\mathrm{sym}}^{(Q)}=1+\frac{1+\eta(q)}{\left(\begin{array}{c}M \\ 2\end{array}\right)} \sum_{i<j}^{M}\left(\left|\tilde{\psi}_{i}^{*} \cdot \tilde{\psi}_{j}\right|^{2}-\sum_{\alpha=1}^{N}\left|\tilde{\psi}_{i}(\alpha) \tilde{\psi}_{j}(\alpha)\right|^{2}\right)$,

where we exploited the normalization $\tilde{\psi}_{i}^{*} \cdot \tilde{\psi}_{j}=\delta_{i, j}$ due to the symmetry condition and the unitarity of $U$. A comparison with Eq. (14) immediately yields

$$
\min \bar{G}_{N=m, M}^{(Q, \mathrm{sym})}=1-\frac{1+\eta(q)}{M} \leq \min \bar{G}_{N=m, M}^{(\mathrm{cl})},
$$

with the minimum value reached by the same optimal interferometer of the classical case, obtained by choosing $U$ to be the $m \times m$ FTM previously defined. This setup can be built in waveguides by using a number of components that scales efficiently with the system dimensionality [49] and was recently proposed as a tool to distinguish real bosonic interference from semiclassical imitations, a problem of interest for the certification of boson sampling [50]. Our findings now show that it can also be adopted to verify the 
impossibility of obtaining the quantum results by means of a classical wavelike model of light. We also note that increasing the system dimensionality reduces the allowed nonclassical range of $\bar{G}$ accessible by symmetric input states, as can be easily observed by comparing Eq. (20) with Eq. (15).

While the classical bound is completely general, the requirement of injecting states in every input port of a quantum interferometer assumes a lossless evolution. However, the same values for $\bar{G}^{(Q)}$ would be obtained in the presence of balanced losses, defined as independent of the path taken by the light in the interferometer. Indeed, their only effect would be the multiplication of the output intensities by a constant efficiency factor, which does not affect the studied correlation function because it simplifies in Eq. (2). Balanced losses can be expected to arise with good approximation if the interferometer is symmetrically built (e.g., see the universal model recently proposed in Ref. [51]).

Interferometer divisibility.-We show how Eq. (20) allows us to develop a sufficient criterion that can certify the impossibility of dividing an interferometer into independent subblocks, therefore certifying "true" $m$-modes interference. Let us consider an interferometer with outputs completely monitored, whose evolution matrix $U$ can be split into two independent submatrices. If $m$ states of light characterized by $\eta \geq 0$ are injected into its input ports, the minimum value achievable by $\bar{G}^{(Q)}$ is obtained when the two subblocks are FTM matrices. Equation (20), and the observation that $\left\langle\hat{I}_{i} \hat{I}_{j}\right\rangle=\left\langle\hat{I}_{i}\right\rangle\left\langle\hat{I}_{j}\right\rangle$ if outputs $i, j$ are taken in different blocks, allow us to write the aforementioned minimum as

$$
\min \bar{G}_{N=m, M=m}^{(Q, \text { sym,div })}=1-(1+\eta) \frac{m-2}{m(m-1)} .
$$

As $\min \bar{G}_{N=m, M=m}^{(Q, \text { sym,div })}$ is strictly larger than the global minimum of Eq. (20) with $M=m \geq 2$, a value of $\bar{G}$ smaller than this threshold will provide the desired certification.

Conclusions. - In this Letter we showed how a normalized quantifier of correlations among pairs of output intensities can yield information on the nonclassicality of the input sources and on the structure of the used multiport interferometer. In particular, we found a tight lower bound for the correlations obtained with a classical setup, where electric fields fully describe the light of the sources. We also discussed the necessity of using sub-Poissonian quantum sources in order to violate this threshold, and characterized the maximal extent of this violation under symmetric input conditions. Our classical bounds confirm the importance of low-order correlation functions in the study of many-particle interference effects. By comparing quantum predictions with classical electromagnetic theory, our results give a new perspective on this fundamental issue and can be of interest for experimentalists as possible tools for characterizing their setups.
We would like to thank W. S. Kolthammer, A. D. K. Plato, and A. Buchleitner for valuable discussions. We acknowledge financial support from the UK EPSRC (EP/ K034480/1), the ERC grant MOQUACINO and the People Programme (Marie Curie Actions) of the EU's Seventh Framework Programme (FP7/2007-2013).

\section{APPENDIX PROOF FOR THE CLASSICAL BOUND}

Here we provide a proof for Eq. (15). First, we prove that the right-hand side is a lower bound, and then that it can be saturated. To do so, it is convenient to formally write the vectors $\psi_{i}$ in Dirac notation: $\psi_{i}(\alpha)=\left\langle\alpha \mid \psi_{i}\right\rangle$. Let then $H=\sum_{i}^{M}\left|\psi_{i}\right\rangle\left\langle\psi_{i}\right|$, and note that the two following inequalities hold:

$\sum_{\alpha=1}^{N}\langle\alpha|H| \alpha\rangle^{2} \leq \operatorname{Tr}\left[H^{2}\right] ; \quad \operatorname{Tr}\left[H^{2}\right] \geq \frac{\operatorname{Tr}[H]^{2}}{\min \{M, N\}}$.

The first can be obtained by using the decomposition of the identity operator $\mathbb{1}=\sum_{\alpha}^{N}|\alpha\rangle\langle\alpha|$ and the properties of the trace. The second follows from the inequality $\operatorname{Tr}\left[\sigma^{2}\right] \geq 1 / \operatorname{rank}(\sigma)$, which applies to any density matrix $\sigma$ because the quantum state with minimal purity is the completely mixed one, by substituting $\sigma=H / \operatorname{Tr}[H]$ and by noticing that $\operatorname{rank}(H) \leq \min \{M, N\}$. Let us now rewrite Eq. (14) as

$$
\begin{aligned}
\bar{G}^{(\mathrm{cl})}= & 1+\frac{1}{M(M-1)}\left(\operatorname{Tr}\left[H^{2}\right]-\sum_{\alpha}^{N}\langle\alpha|H| \alpha\rangle^{2}\right. \\
& \left.-M+M \sum_{\alpha=1}^{N} \sum_{i=1}^{M} \frac{1}{M}\left(\left\langle\alpha\left|\left[\left|\psi_{i}\right\rangle\left\langle\psi_{i}\right|\right]\right| \alpha\right\rangle\right)^{2}\right) .
\end{aligned}
$$

The convexity of the square function allows us to lower bound the last term between brackets with $\sum_{\alpha=1}^{N}\langle\alpha|H| \alpha\rangle^{2} / M$. At this stage, the application of the two inequalities given in Eq. (A1) (in the order in which they appear) leads to the desired lower bound. Its tightness can be easily proven by considering

$$
\left\langle\alpha \mid \psi_{i}\right\rangle= \begin{cases}\frac{1}{\sqrt{\min \{M, N\}}} \omega_{M}^{(i-1)(\alpha-1)} & \text { if } \alpha \leq M \\ 0 & \text { if } \alpha>M .\end{cases}
$$

*1.rigovacca14@imperial.ac.uk

[1] C. K. Hong, Z. Y. Ou, and L. Mandel, Phys. Rev. Lett. 59, 2044 (1987).

[2] F. W. Sun and C. W. Wong, Phys. Rev. A 79, 013824 (2009).

[3] E. Knill, R. Laflamme, and G. J. Milburn, Nature (London) 409, 46 (2001).

[4] Y. H. Shih and C. O. Alley, Phys. Rev. Lett. 61, 2921 (1988). 
[5] M. Halder, A. Beveratos, N. Gisin, V. Scarani, C. Simon, and H. Zbinden, Nat. Phys. 3, 692 (2007).

[6] B. C. Sanders, Phys. Rev. A 40, 2417 (1989).

[7] H. Lee, P. Kok, and J. P. Dowling, J. Mod. Opt. 49, 2325 (2002).

[8] V. Giovannetti, S. Lloyd, and L. Maccone, Nat. Photonics 5, 222 (2011).

[9] R. Demkowicz-Dobrzański, M. Jarzyna, and J. Kołodyński, in Quantum Limits in Optical Interferometry, Progress in Optics, Vol. 60, edited by E. Wolf (Elsevier, New York, 2015), Chap. 4, pp. 345-435.

[10] L. Mandel, Phys. Rev. A 28, 929 (1983).

[11] H. Paul, Rev. Mod. Phys. 58, 209 (1986).

[12] M. C. Tichy, M. Tiersch, F. de Melo, F. Mintert, and A. Buchleitner, Phys. Rev. Lett. 104, 220405 (2010).

[13] Y. L. Lim and A. Beige, New J. Phys. 7, 155 (2005).

[14] R. Lopes, A. Imanaliev, A. Aspect, M. Cheneau, D. Boiron, and C. I. Westbrook, Nature (London) 520, 66 (2015).

[15] K. Toyoda, R. Hiji, A. Noguchi, and S. Urabe, Nature (London) 527, 74 (2015).

[16] M. Jachura and R. Chrapkiewicz, Opt. Lett. 40, 1540 (2015).

[17] D. Sokolovski, J. Siewert, and L. M. Baskin, Phys. Rev. A 93, 012705 (2016).

[18] J. D. Urbina, J. Kuipers, S. Matsumoto, Q. Hummel, and K. Richter, Phys. Rev. Lett. 116, 100401 (2016).

[19] J. B. Spring, P. L. Mennea, B. J. Metcalf, P. C. Humphreys, J. C. Gates, H. L. Rogers, C. Soeller, B. J. Smith, W. S. Kolthammer, P. G. R. Smith, and I. A. Walmsley, arXiv: 1603.06984

[20] M. Tillmann, S. H. Tan, S. E. Stoeckl, B. C. Sanders, H. de Guise, R. Heilmann, S. Nolte, A. Szameit, and P. Walther, Phys. Rev. X 5, 041015 (2015).

[21] N. Spagnolo, C. Vitelli, L. Aparo, P. Mataloni, F. Sciarrino, A. Crespi, R. Ramponi, and R. Osellame, Nat. Commun. 4, 1606 (2013).

[22] B. J. Metcalf, N. Thomas-Peter, J. B. Spring, D. Kundys, M. A. Broome, P. C. Humphreys, X.-M. Jin, M. Barbieri, W. S. Kolthammer, J. C. Gates, B. J. Smith, N. K. Langford, P. G. Smith, and I. A. Walmsley, Nat. Commun. 4, 1356 (2013).

[23] R. Crespi, A. Osellame, R. Ramponi, D. J. Brod, E. F. Galvao, N. Spagnolo, C. Vitelli, E. Maiorino, P. Mataloni, and F. Sciarrino, Nat. Photonics 7, 545 (2013).

[24] J. B. Spring, B. J. Metcalf, P. C. Humphreys, W. S. Kolthammer, X.-M. Jin, M. Barbieri, A. Datta, N. Thomas-Peter, N. K. Langford, D. Kundys, J. C. Gates, B. J. Smith, P. G. R. Smith, and I. A. Walmsley, Science 339, 798 (2013).

[25] X. C. Yao, T. X. Wang, P. Xu, H. Lu, G. S. Pan, X. H. Bao, C. Z. Peng, C. Y. Lu, Y.A. Chen, and J. W. Pan, Nat. Photonics 6, 225 (2012).

[26] J. C. F. Matthews, A. Politi, D. Bonneau, and J. L. O'Brien, Phys. Rev. Lett. 107, 163602 (2011).

[27] S. Aaronson and A. Arkhipov, in Proceedings of the FortyThird Annual ACM Symposium on Theory of Computing (STOC '11), (ACM, New York, 2011), pp. 333-342.

[28] V. S. Shchesnovich, Phys. Rev. Lett. 116, 123601 (2016).

[29] M. Bentivegna, N. Spagnolo, C. Vitelli, F. Flamini, N. Viggianiello, L. Latmiral, P. Mataloni, D. J. Brod,
E. F. Galvão, A. Crespi, R. Ramponi, R. Osellame, and F. Sciarrino, Sci. Adv. 1, e1400255 (2015).

[30] M. A. Broome, A. Fedrizzi, S. Rahimi-Keshari, J. Dove, S. Aaronson, T. C. Ralph, and A. G. White, Science 339, 794 (2013).

[31] N. Spagnolo, C. Vitelli, M. Bentivegna, D. J. Brod, A. Crespi, F. Flamini, S. Giacomini, G. Milani, R. Ramponi, P. Mataloni, R. Osellame, E. F. Galvao, and F. Sciarrino, Nat. Photonics 8, 615 (2014).

[32] M. C. Tichy, M. Tiersch, F. Mintert, and A. Buchleitner, New J. Phys. 14, 093015 (2012).

[33] A. V. Belinskii and D. N. Klyshko, Sov. Phys. JETP 75, 606 (1992).

[34] K. Mayer, M. C. Tichy, F. Mintert, T. Konrad, and A. Buchleitner, Phys. Rev. A 83, 062307 (2011).

[35] S. Laibacher and V. Tamma, Phys. Rev. Lett. 115, 243605 (2015).

[36] M. C. Tichy, H. T. Lim, Y. S. Ra, F. Mintert, Y. H. Kim, and A. Buchleitner, Phys. Rev. A 83, 062111 (2011).

[37] Y. Ra, M. C. Tichy, H.-T. Lim, O. Kwon, F. Mintert, A. Buchleitner, and Y. Kim, Proc. Natl. Acad. Sci. U.S.A. 110, 1227 (2013).

[38] V. Tamma and S. Laibacher, Phys. Rev. Lett. 114, 243601 (2015).

[39] C. Thiel, T. Bastin, J. Martin, E. Solano, J. von Zanthier, and G. S. Agarwal, Phys. Rev. Lett. 99, 133603 (2007).

[40] S. Oppel, T. Büttner, P. Kok, and J. von Zanthier, Phys. Rev. Lett. 109, 233603 (2012).

[41] D. Gatto Monticone, K. Katamadze, P. Traina, E. Moreva, J. Forneris, I. Ruo-Berchera, P. Olivero, I. P. Degiovanni, G. Brida, and M. Genovese, Phys. Rev. Lett. 113, 143602 (2014).

[42] M. E. Pearce, T. Mehringer, J. von Zanthier, and P. Kok, Phys. Rev. A 92, 043831 (2015).

[43] M. Walschaers, J. Kuipers, J. Urbina, K. Mayer, M. C. Tichy, K. Richter, and A. Buchleitner, New J. Phys. 18, 032001 (2016).

[44] M. Walschaers, J. Kuipers, and A. Buchleitner, Phys. Rev. 94, 020104(R) (2016).

[45] K. J. Blow, R. Loudon, S. J. D. Phoenix, and T. J. Shepherd, Phys. Rev. A 42, 4102 (1990).

[46] See Supplemental Material at http://link.aps.org/ supplemental/10.1103/PhysRevLett.117.213602 for the optimality proof of the considered classical setup and for general expressions of intensity correlations in the quantum framework.

[47] For the sake of completeness, the effective probability distribution $q_{\alpha}\left(n_{\alpha}\right)$ is related to $p_{\alpha}\left(\xi_{\alpha}\right)$ through the relation $q_{\alpha}\left(n_{\alpha}\right)=\sum_{\xi_{\alpha}} p_{\alpha}\left(\xi_{\alpha}\right) n_{\alpha} !\left|\varphi_{\alpha}^{\left(\xi_{\alpha}\right)}\left(n_{\alpha}\right)\right|^{2}$.

[48] Z. Y. Ou, Phys. Rev. A 37, 1607 (1988).

[49] A. Crespi, R. Osellame, R. Ramponi, M. Bentivegna, F. Flamini, N. Spagnolo, N. Viggianiello, L. Innocenti, P. Mataloni, and F. Sciarrino, Nat. Commun. 7, 10469 (2016).

[50] M. C. Tichy, K. Mayer, A. Buchleitner, and K. Mølmer, Phys. Rev. Lett. 113, 020502 (2014).

[51] W. R. Clements, P. C. Humphreys, B. J. Metcalf, W. S. Kolthammer, and I. A. Walmsley, arXiv:1603.08788. 